\title{
Enfermedades del estomago y duodeno en imagenes
}

Pr J. FERRANDO - Editorial Médica Internacional, SA Madrid 1992

(sponsor GLAXO)

Le Professeur J. FERRANDO de l'Université de Valence a récidivé en réalisant un ouvrage d'endoscopies digestives intéressant l'estomac et le duodénum. On retrouve la même qualité que lors du précédent volume, intéressant l'cesophage, associant une iconographie entièrement faite d'images d'endoscopie électronique avec en regard les schémas explicatifs des lésions constatées.

Les chapitres envisagés concernent l'aspect endoscopique normal de l'œsophage, les hernies hiatales, les gastrites chroniques et aiguës, des maladies moins fréquentes sont décrites avec de belles images endoscopiques: tuberculose gastrique, gastro-entérite éosinophile, maladie de Cröhn, maladie de ménétrier sans oublier les multiples aspects que réalisent les lymphomes gastriques.

Sont envisagés ensuite les ulcères gastriques dans leurs différentes variétés bénins, malins avec un développement imagé sur les phénomènes de cicatrisation. Sont également décrits en image les multiples aspects que peuvent réaliser les polypes hyperplasiques, les adénomes villeux, les adénomes tubulaires et les tumeurs d'origine mésenchymateuse ou comme celles d'origine hétérotypiques : tératome, pancréas aberrant. Le problème posé par les cancers gastriques est largement détaillé puisqu'il couvre 20 pages avec de belles illustrations de tumeurs moins fréquentes telles que les carcinoïdes gastriques ;

Les pièges endoscopiques par l'estomac opéré sont bien illustrés pour toutes les interventions, de même que sont envisagées les pathologies liées à cet estomac opéré : carcinome sur fil de suture, ulcère de la bouche anastomotique, gastrite du moignon, formation de bézoards, cancer du moignon et sténose de l'anastomose.

Tout un chapitre est réservé à l'extraction de corps étrangers, là encore avec de magnifiques vues d'endoscopie électronique. Enfin pour conclure la pathologie gastrique sont décrites les anomalies vasculaires: varices, télangiectasies s'intégrant dans le cadre de la Maladie de Rendu osler, ectasies vasculaires de l'antre.

On retrouve la même qualité d'images pour ce qui concerne la pathologie duodénale qu'il s'agisse de duodénites spécifiques ou non spécifiques de localisation duodénale, maladie de Cröhn.

Les clichés de maladie coeliaque sont peut-être un peu surexposés avec un effet «blooming "; par contre tout ce qui concerne la pathologie ulcéreuse du cadre duodénal est remarquablement bien fait, de même tout ce qui concerne les tumeurs bénignes, malignes du duodénum ainsi que la description de diverticules et de ce qu'entraînent les compressions extrinsèques.

Au total il s'agit d'un livre de qualité qui fait honneur à l'Ecole de gastro-entérologie espagnole, il nous paraît complet. La qualité des clichés sur le plan endoscopique est encore meilleur que lors du premier livre. Cet ouvrage est à conseiller aux lecteurs français qui comprennent l'espagnol, mais aussi à ceux qui ne pratiquent pas la langue de Cervantès car les schémas qui viennent souligner les photos endoscopiques suffisent comme explication.

Gérard GAY

\section{International Conference on Cellular and Molecular Aspects of Self-reactivity and Autoimmune Disorders}

\author{
Taormina, Italy - June 7-12, 1992
}

Le système immunitaire des mammifères génère au hasard des lymphocytes $T$ et $B$ spécifiques d'antigènes. Ainsi l'organisme, grâce à ces cellules hautement spécialisées, pourra reconnaître tout agent infectieux étranger et l'éliminer grâce à une réponse immune appropriée. Comme ces récepteurs sont produits au hasard, une partie du réper- toire immunitaire est théoriquement capable de reconnaître les antigènes du soi. Des mécanismes de sélection sont donc nécessaires pour favoriser le développement des lymphocytes bénéfiques à la défense de l'individu et pour détruire ou rendre inefficaces les lymphocytes auto-réactifs. Si ces mécanismes sont insuffisants ou inappropriés, 